\title{
Fatores associados a quedas em idosos socorridos pelo Serviço de Atendimento Móvel de Urgência - SAMU 192
}

Thais Kellen Ribeiro da Silva ${ }^{1}$ Isadora dos Reis Martins ${ }^{1}$, Johann Peter Amaral dos Santos ${ }^{1}$, Leonardo França Vieira ${ }^{2}$, Caio Duarte Neto ${ }^{3}$, Luciana Carrupt Machado Sogame ${ }^{4 *}$

${ }^{1}$ Graduandos de Medicina da EMESCAM

${ }^{2}$ Enfermeiro especialista em Emergência. Mestre em Políticas Públicas e Desenvolvimento Local pela EMESCAM.

Docente dos Cursos de Graduação em Enfermagem, Medicina e Fisioterapia da EMESCAM.

${ }^{3}$ Médico, cirurgião geral, especialista em Medicina de Emergência e Cirurgia do Trauma. Mestre em Políticas Públicas e Desenvolvimento Local pela EMESCAM. Docente do Curso de Graduação de Medicina da EMESCAM.

${ }^{4}$ Fisioterapeuta. Doutora em Ciências pela Unifesp. Docente dos Cursos de Graduação em Fisioterapia e Medicina.

Docente do quadro permanente de Mestrado em Políticas Públicas e Desenvolvimento Local da EMESCAM.

*Autor correspondente. Endereço de e-mail: luciana.sogame@emescam.br

\section{RESUMO}

A queda é o principal evento traumático na população geriátrica. Objetivo: Verificar a prevalência e os fatores associados a quedas de idosos socorridos pelo SAMU. Método: Estudo transversal com coleta retrospectiva de informações de 861 pacientes idosos, a partir de amostra sistemática dos Boletins de Ocorrência dos Atendimentos Pré-Hospitalares do Serviço de Atendimento Móvel de Urgência (SAMU 192), no estado do Espírito Santo, em 2015. Coletaram-se informações quanto: idade, sexo, região de domicílio, do atendimento (período da semana, turno da solicitação, gravidade presumida pelo Médico Regulador, gravidade real, tipo de recurso enviado e desfecho da ocorrência). Foram realizados o Teste Qui-quadrado e o Resíduo do Qui-quadrado. Resultados: A prevalência de quedas foi de 14,6\%, sendo mais comum a queda da própria altura (78,6\%). As vítimas possuíam, em sua maioria, de 60 a 79 anos $(65,7 \%)$ e eram do sexo feminino $(51,7 \%)$, foram atendidos durante a semana $(71,9 \%)$, no turno diurno $(63,5 \%)$, apresentaram classificação amarela $(57,1 \%)$, tiveram maior risco $(51,3 \%)$, receberam o auxílio do suporte básico $(74,6 \%)$ e foram transportados para um serviço de saúde $(71,4 \%)$. Os fatores associados às quedas $(p<0,001)$ foram a classificação amarela, menor risco de gravidade real, envio de suporte básico e transporte ao final do atendimento. Conclusão: A prevalência de quedas foi de 14,6\%. 0 perfil dos idosos vítimas de quedas mostrou-se idêntico aos que foram atendidos por outras causas. Entretanto, comportaram-se como fatores associados à queda: menor risco na gravidade real, gravidade presumida amarela, envio de suporte básico e transporte para serviço de saúde.

Palavras-chave: Idoso. Acidentes por quedas. Serviços médicos de emergência.

\section{Introdução}

Na população geriátrica, a queda é o principal evento traumático quanto à morbidade e mortalidade, mesmo quando envolve baixa energia cinética, uma vez que os idosos possuem menor reserva fisiológica e resposta frente à agressão, em decorrência do processo de envelhecimento ${ }^{1}$. Neste cenário, é crescente e notório o número de internações e óbitos em decorrência desse tipo de evento, cuja frequência é maior com o avançar da idade ${ }^{2}$. Contudo, apesar de se tratar de um tema relevante nesta população, poucos são os estudos encontrados sobre a temática no âmbito do atendimento pré-hospitalar móvel, fato observado em revisão integrativa sobre os serviços de saúde que atendem vítimas de violência, onde se verificou que apenas 6 das 304 pesquisas eram sobre atendimento pré-hospitalar móvel e fixo. Destaca-se 
que, desses, apenas 2 foram realizados na população idosa $^{3}$.

No Brasil, o atendimento pré-hospitalar móvel à vítima em situação de urgência ou emergência é realizado pelo SAMU 192, o qual é o componente de atendimento pré-hospitalar do sistema público de saúde, criado pelo Ministério da Saúde no ano de 2003 e estabelecido em todo o território nacional ${ }^{5}$. No Espírito Santo, em 2015, 82,3\% das causas externas em idosos atendidos pelo Serviço de Atendimento Móvel de Urgência (SAMU 192/ES) foram decorrentes de quedas, demonstrando a maior suscetibilidade dessa população a esse tipo de ocorrência ${ }^{4}$.

Em relação aos custos empregados a esse grupo etário em decorrência das quedas, verifica-se que não se restringem apenas às hospitalizações, mas estendem-se à reabilitação, às medicações e ao tratamento médico oneroso dos serviços de saúde, gerando fortes pressões a esse sistema ${ }^{6}$. Segundo Dhalwani et al. ${ }^{7}$, na Europa, $5 \%$ das quedas resultam em fraturas e as lesões decorrentes desse trauma são a quinta maior causa de morte em idosos, correspondendo a um grande número de internações a um custo de 2,3 bilhões de euros por ano.

No Brasil, cerca de $30 \%$ dos idosos sofrem quedas no período de um $\mathrm{ano}^{8}$. No âmbito do Sistema Único de Saúde (SUS), entre 2000 e 2018, as hospitalizações por quedas e custos correspondentes aumentaram, chegando a um gasto de, aproximadamente, 195 milhões em 2018, o que corresponde a um acréscimo em torno de $73 \%$ em relação ao ano $2000^{8}$. De acordo com a literatura, entre os fatores associados à queda da população idosa estão: sexo feminino, possuir 80 anos ou mais, maior número de morbidades, polifarmácia e período diurno ${ }^{9,10}$.

A realização de estudos que evidenciem as características dos idosos vítimas de queda oriundos da assistência pré-hospitalar móvel faz-se, portanto, necessária, para que se identifique os fatores associados e seus consequentes agravos, a fim de melhorar o serviço prestado, uma vez que a literatura abrangendo idosos no serviço pré-hospitalar é reduzida e por vezes não compreende as variáveis abordadas no presente estudo.

Diante do exposto, o presente estudo teve por objetivo verificar a prevalência e os fatores associados a quedas de idosos socorridos pelo SAMU 192 do estado do Espírito Santo.

\section{Métodos}

Trata-se de um estudo transversal a partir da coleta retrospectiva de dados dos Boletins de Ocorrência (BO) impressos dos Atendimentos PréHospitalares do SAMU 192, do estado do Espírito Santo. A pesquisa foi aprovada pelo Comitê de Ética em Pesquisa da Escola Superior de Ciências da Santa Casa de Misericórdia de Vitória, por constituir parte dos objetivos da pesquisa "Rede de Urgência e Emergência: Estudo do SAMU 192 na Região Metropolitana do ES", aprovada por meio do Parecer no 1.748 .503 .

No ano de 2015, o SAMU 192 realizou 51.555 atendimentos no Espírito Santo. Para a definição da quantidade de participantes da pesquisa, considerouse a seguinte fórmula: $n 0=Z 2 \times \delta 2 /$ e2, em que n0 é o número mínimo amostral, Z2 o valor correspondente ao intervalo de confiança (IC) de $95 \%$, $\delta 2(\delta=1 / 2$, ou seja, 0,5$)$ a proporção que se espera encontrar e E2 o erro amostral tolerado $(E=0,05)$. Assim, o número amostral mínimo obtido foi de 2.401, sendo, ao final, arredondado para 2.500, a fim de minimizar os riscos de erro e perda amostrais. Para a seleção, aplicou-se o método de amostragem sistemática com intervalo de seleção preestabelecido, em que a cada vinte ocorrências, a vigésima foi selecionada para compor a pesquisa. A amostra coletada contém um total de 2.502 atendimentos primários feitos pelo SAMU 192.

Coletaram-se, por meio de ficha padrão, informações sociodemográficas e do atendimento dos 861 idosos ( $\geq 60$ anos), que receberam atendimento primário realizado pelo SAMU 192, no ano de 2015. Quanto às questões sociodemográficas, interessaram idade (60 a 79 anos e maior ou igual a 80 anos), sexo (feminino e masculino) e região de domicílio (Região de Vitória, Região de Guarapari, Região de Santa Teresa, Região de Venda Nova do Imigrante). Os municípios foram agrupados nessas categorias para fins de análise, seguindo a logística administrativa do SAMU 192 e sua área de abrangência, considerando no mínimo uma Unidade de Suporte Avançado (USA) por região.

Sobre o atendimento, foram coletados: período da semana (segunda a sexta-feira e fim de semana), turno da solicitação (diurno e noturno), tipo de recurso enviado (Suporte Básico ou Suporte Avançado), tipo de ocorrência (clínica, traumática, psiquiátrica e ginecológica), gravidade presumida pelo Médico Regulador (vermelho, amarelo e verde/azul), a gravidade real (maior e menor risco), e 
- desfecho da ocorrência (transportado e não transportado para serviço de saúde).

Quanto aos recursos enviados, o Suporte Básico é empregado em casos de menor complexidade e gravidade, visto que, nesses tipos de atendimento, o mecanismo de trauma envolve menor energia e exige apenas um técnico de enfermagem ou auxiliar de enfermagem e motorista. Por sua vez, o Suporte Avançado é acionado para casos mais graves e conta com um médico, um enfermeiro, o motorista e maiores recursos para possíveis intervenções ${ }^{11}$.

Para fins de análise estatística, a variável dependente foi categorizada em quedas, distinta em queda da própria altura, de altura menor que seis metros ou maior que seis metros, e outras ocorrências. A gravidade presumida pelo Médico Regulador representa a classificação quanto ao nível de urgência/gravidade estimada no momento do acionamento, categorizada como: Prioridade Absoluta (vermelho), compreendendo os casos em que há risco iminente de vida e/ou perda funcional grave, imediata ou secundária; Prioridade Moderada (amarelo), determinada quando 0 atendimento médico é exigido dentro de poucas horas; Prioridade Baixa (Verde), que inclui casos com necessidade de avaliação médica, sem risco de vida ou perda funcional, podendo aguardar várias horas; e Prioridade Mínima (azul), nas situações em que orientações pelo telefone podem ser realizadas pelo Regulador, bem como encaminhamentos ${ }^{12}$. A gravidade real foi mensurada pelo score q-Sofa, que considera como parâmetros pressão arterial sistólica abaixo de $100 \mathrm{mmHg}$, frequência respiratória maior que 22 irpm e alteração do estado mental (Escala de Coma de Glasgow <15). Os resultados foram agrupados em menor risco, quando score $=0$, e maior risco para score $\geq 1$.

As informações obtidas foram analisadas por meio de estatística descritiva simples, com os cálculos de frequência, média, valores mínimos e máximos, bem como desvio padrão. A associação entre as variáveis independentes e o desfecho primário foi feita a partir do Teste Qui-Quadrado ou, no caso de frequências esperadas inferiores a cinco, - Exato de Fisher. As variáveis consideradas independentes foram as sociodemográficas (idade, sexo e região de domicílio) e as variáveis do atendimento (período da semana, turno de solicitação e tipo de ocorrência, tipo de recurso enviado, gravidade real, gravidade presumida e desfecho da ocorrência) e, como variável dependente, a presença de queda. Adotou-se o nível de significância de 5\% $(p<0,05)$, com Intervalo de Confiança de 95\% (IC95\%). O Resíduo do Qui-Quadrado (resíduo ajustado) foi calculado para as variáveis com significância estatística $(p<0,05)$, considerando-se associação significante entre duas categorias quando o resíduo foi maior que 1,96.

\section{Resultados}

Dos 2.502 atendimentos estudados, 861 $(34,4 \%)$ foram a idosos. A prevalência de quedas foi de $14,6 \%$. Ao considerar o tipo de ocorrência, verificou-se que a prevalência de ocorrências de caráter clínico foi de $80,8 \%$, enquanto $17,7 \%$ foram traumáticas e outros $1,4 \%$ corresponderam às psiquiátricas. Das ocorrências traumáticas, 82,3\% foram decorrentes de quedas, sendo $78,6 \%$ vítimas de queda da própria altura, $20,6 \%$ de altura menor que 6 metros e $0,8 \%$ sem especificação do tipo de queda. A média de idade dos pacientes foi $75( \pm 9,9$ anos), variando de 60 a 110 anos.

Os idosos atendidos pelo SAMU 192 possuíam, em sua maioria, de 60 a 79 anos (65,7\%) e eram do sexo feminino $(51,7 \%)$. Eles foram atendidos durante a semana $(71,9 \%)$ e no turno diurno $(63,5 \%)$. Estavam domiciliados na região de Vitória $(83,6 \%)$, classificados como prioridade moderada (amarelo) $(57,1 \%)$, tiveram maior risco $(51,3 \%)$, receberam o auxílio do suporte básico $(74,6 \%)$ e foram transportados para um serviço de saúde $(71,4 \%)$.

A Tabela 1 apresenta 0 resultado da comparação entre as características dos idosos vítimas de queda e os atendidos por outras ocorrências.

\begin{tabular}{lccc}
\hline \hline \multicolumn{1}{c}{ VARIÁVEIS } & $\begin{array}{c}\text { QUEDA } \\
\text { N= 126 }\end{array}$ & $\begin{array}{c}\text { OUTRAS } \\
\text { OCORRÊNCIAS } \\
\mathbf{N}=735\end{array}$ & $\boldsymbol{p}^{*}$ \\
\hline \hline & $\mathrm{n}(\%)$ & $\mathrm{n}(\%)$ & \\
\hline Idade & $83(65,9)$ & $483(65,7)$ & 0,972 \\
$60-79$ anos & $43(34,1)$ & $252(34,3)$ & \\
$\geq 80$ anos & $65(51,6)$ & $380(51,7)$ & 0,981 \\
$\begin{array}{l}\text { Sexo } \\
\text { Feminino }\end{array}$ & $61(48,4)$ & $355(48,3)$ & \\
Masculino & $91(72,2)$ & $528(71,8)$ & \\
$\begin{array}{l}\text { Período da semana } \\
\text { Segunda a sexta }\end{array}$ & $34(27)$ & $203(27,6)$ & 0,895 \\
Final de semana & $1(0,8)$ & $4(0,5)$ & \\
Não informado & $83(65,9)$ & $464(63,1)$ & 0,474 \\
\hline Turno da solicitação & &
\end{tabular}




\begin{tabular}{|c|c|c|c|}
\hline Noturno & $31(24,6)$ & $172(23,4)$ & \multirow{7}{*}{0,900} \\
\hline Não informado & $12(9,5)$ & $99(13,5)$ & \\
\hline \multicolumn{4}{|l|}{ Região da ocorrência } \\
\hline Grande Vitória & $102(80,9)$ & $618(84,1)$ & \\
\hline Guarapari & $9(7,1)$ & $42(5,7)$ & \\
\hline $\begin{array}{l}\text { Venda Nova do } \\
\text { Imigrante }\end{array}$ & $4(3,2)$ & $22(3,0)$ & \\
\hline Santa Teresa & $4(3,2)$ & $28(3,8)$ & \\
\hline Não informado & $7(5,6)$ & $25(3,4)$ & \\
\hline \multicolumn{3}{|l|}{ Gravidade presumida } & \multirow{4}{*}{$<0,001$} \\
\hline Amarelo & $92^{\dagger}(73)$ & $400(54,4)$ & \\
\hline Verde/azul & $10(7,9)$ & $63(8,6)$ & \\
\hline Vermelho & $12(9,5)$ & $172^{\dagger}(23,4)$ & \\
\hline \multicolumn{3}{|l|}{ Gravidade real } & \multirow{3}{*}{$<0,001$} \\
\hline Maior risco & $36(28,6)$ & $406^{\dagger}(55,2)$ & \\
\hline Menor risco & $90^{\dagger}(71,4)$ & $329(44,8)$ & \\
\hline \multicolumn{3}{|l|}{ Recurso enviado } & \multirow{4}{*}{$<0,001$} \\
\hline Suporte Básico & $\begin{array}{c}110^{\dagger} \\
(87,3)\end{array}$ & $533(72,5)$ & \\
\hline Suporte Avançado & $5(4,0)$ & $162^{\dagger}(22,0)$ & \\
\hline Não informado & $11(8,7)$ & $40(5,4)$ & \\
\hline \multicolumn{3}{|l|}{$\begin{array}{l}\text { Desfecho da } \\
\text { ocorrência }\end{array}$} & \multirow{4}{*}{$<0,001$} \\
\hline Transportado & $\begin{array}{c}113^{\dagger} \\
(89,7)\end{array}$ & $502(68,3)$ & \\
\hline Não transportado & $11(8,7)$ & $224^{\dagger}(30,5)$ & \\
\hline Não informado & $2(1,6)$ & $9(1,2)$ & \\
\hline Teste Qui-quadrado & & & \\
\hline Resíduo maior que 1,96 & & & \\
\hline
\end{tabular}

Tabela 1. Características dos idosos vítimas de quedas e outras ocorrências socorridos pelo SAMU 192. Vitória, ES, 2015.

\section{Discussão}

O principal motivo de atendimento a idosos atendidos pelo SAMU 192/ES é decorrente principalmente de evento clínico. Em sua maioria, eles tinham de 60 a 79 anos, eram do sexo feminino e foram atendidos durante a semana e no turno diurno. Não se verificou diferença entre os pacientes vítimas de quedas e de outras causas, que seguiram o perfil geral. Comportaram-se como fatores associados à queda um menor risco na gravidade real, a gravidade estimada amarela, o envio de suporte básico e o transporte para serviço de saúde.

No que se refere à idade dos idosos atendidos, o predomínio da faixa etária de 60-79 anos era esperado, uma vez que essa parcela da população é maior do que a de 80 anos ou mais ${ }^{13}$.
Além disso, o dado corrobora com os resultados de estudo desenvolvido por Esteves et $\mathrm{al}^{14}$, que descreve o perfil dos idosos atendidos pelo SAMU 192, na cidade de Juiz de Fora (MG) e aponta para maior prevalência de idosos com idade entre 60 e 79 anos. Assim, a ocorrência de quedas ou quaisquer outras intercorrências em idosos é mais frequente naqueles que são mais jovens, mas há de se considerar o tamanho dessa faixa etária na população brasileira em detrimento dos idosos com 80 anos ou mais.

Além disso, ao ponderar isoladamente sobre cada faixa etária, nota-se que há diferença com maior prevalência das quedas em idosos que possuíam 80 anos ou mais. $O$ dado converge com os das pesquisas de Franck et $\mathrm{al}^{9}$ e Moreland B, Kakara R, Henry A. ${ }^{15}$ que observaram maior prevalência de quedas em idosos residentes de comunidades do Brasil e na população dos Estados Unidos. Tal evento ocorre devido a algumas condições inerentes ao envelhecimento, como a perda de densidade óssea, instabilidade postural e diminuição da acuidade visual, que predispõem à queda ${ }^{16,17}$. Esse fato pode estar associado à maior exposição dos idosos entre 60-79 anos aos fatores externos, o que aumenta o risco de quedas ${ }^{18}$. Ressalta-se, contudo, que, na população de idosos estudada, não foi encontrada significância estatística.

Quanto ao sexo, não houve grande diferença percentual em ambos os grupos. Contudo, estudos semelhantes sobre quedas em idosos apontam para o predomínio do sexo feminino ${ }^{19,20,21}$, algo esperado quando se considera que as mulheres apresentam maior expectativa de vida e, com isso, maior exposição às ocorrências diversas ${ }^{9}$. Soma-se a isso os fatores hormonais, além da maior perda de massa magra e força muscular em relação aos homens ${ }^{22}$.

Não foi encontrada diferença entre a ocorrência de quedas e outros atendimentos nos diferentes períodos da semana, quando comparados proporcionalmente. Franklin et al. ${ }^{17}$, afirmam, porém, que $72,7 \%$ das quedas sofridas por idosos ocorrem em dias úteis, quando se dá a maior realização de atividades. Além disso, a predominância das solicitações no período diurno foi constatada, convergindo com outros trabalhos encontrados na literatura ${ }^{23,24}$. Tal fato também pode estar associado à maior realização de tarefas cotidianas no turno diurno $^{25}$ e pode, ainda, ter relação com a maior disponibilidade de acompanhantes neste horário, o que facilitaria a solicitação do serviço ${ }^{24}$.

A região de Vitória compreende os municípios de Cariacica, Serra, Viana, Vila Velha, Vitória e 
concentrava no ano de 2010, aproximadamente, $44,5 \%$ de toda a população do estado do Espírito Santo ${ }^{26}$. Dessa forma, o maior número de atendimentos tanto aos idosos vítimas de queda, quanto aos idosos com outras ocorrências nessa região justifica-se pela concentração populacional.

Ao associar a gravidade estimada ao tipo de ocorrência atendida, observou-se que a maioria dos idosos com quedas tiveram a classificação amarela, que aponta para o risco de esses idosos sofrerem consequências classificadas como prioridade moderada pelo Médico Regulador. Além disso, a predominância dessa classificação indica o correto processo de regulação, uma vez que a queda constitui um trauma de baixa energia e não leva a danos graves de imediato ${ }^{27}$. Entretanto, devido à maior fragilidade do idoso, há maior frequência de internação hospitalar e consumo de recursos dos serviços de saúde, visto que os idosos apresentam maior propensão a lesões mais graves, apesar da baixa gravidade do trauma ${ }^{28}$. Segundo Costa e Fortes $^{28}, 5$ a $25 \%$ das quedas podem causar lesões relevantes, sendo as fraturas, nesse grupo etário, derivadas por traumas de baixa energia.

Em idosos atendidos por outros eventos, a classificação vermelha foi influenciada pelo tipo de ocorrência, indicando que idosos atendidos por causas clínicas, psiquiátricas, traumáticas - que incluem os acidentes de transporte terrestre, excetuando-se quedas -, têm risco de danos mais graves e de prioridade absoluta, de acordo com a regulação médica. Isso pode ocorrer devido a outros mecanismos do trauma de maior energia e às situações clínicas que são mais incapacitantes e letais na população idosa, especialmente as de origem cardiovascular, ao se considerar as condições clínicas e os acidentes de transporte terrestre no trauma ${ }^{17,27}$

Em relação ao recurso enviado aos atendimentos, os resultados dessa pesquisa são semelhantes aos achados de outros estudos, nos quais o Suporte Básico foi a responsável por $85 \%$ dos atendimentos ${ }^{17,27}$. Sendo assim, tais dados demonstram uma consonância com a gravidade estimada, sendo majoritariamente das cores verde/azul ou amarelo com o envio do Suporte Básico como recurso mais enviado ${ }^{27}$, estatisticamente significante nos idosos vítimas de quedas. Nos atendimentos ao idosos sem quedas, também se observou predomínio do Suporte Básico, entretanto, houve associação do envio do Suporte Avançado, o que pode se relacionar à maior gravidade do paciente, apontada pela análise inferencial como presumidamente vermelha e de maior risco nos pacientes sem quedas.

Referente ao desfecho das ocorrências, verificou-se associação dos idosos que sofreram quedas com transporte para algum serviço de saúde. Dados concordantes foram observados nos estudos de Silva et al. ${ }^{27}$ em que $91,2 \%$ dos idosos foram transportados, o que demonstra a importância do transporte para tratamento definitivo da vítima. Nos idosos não acometidos por quedas, notou-se associação significante com idosos não transportados. Tal dado pode estar relacionado aos óbitos e liberações no local, bem como às recusas ao atendimento, resultados encontrados também por Silva et al. ${ }^{27}$

Quanto ao tipo de queda, os achados desse estudo corroboram com os dados na literatura, sendo semelhantes aos estudos de Franklin et al. ${ }^{17}$ e Guerra et al. ${ }^{18}$, nos quais foi observada a prevalência de queda da própria altura de $85,8 \%$ e $87,5 \%$, respectivamente. Além das razões já discutidas, como perda de força muscular e diminuição da acuidade visual, esse tipo de queda pode estar relacionado ao ambiente inadequado em que o idoso está inserido, tanto no domicílio com a presença de pisos escorregadios quanto em vias públicas, uma vez que grande parte dos locais públicos não são devidamente adaptados ${ }^{2,18}$.

Por fim, é fundamental oferecer uma assistência eficaz no ambiente pré-hospitalar, visto que os idosos possuem uma resposta fisiológica limitada às lesões do trauma e muitas vezes uma apresentação atípica do quadro clínico. Por isso, se faz necessário a capacitação dos profissionais do SAMU, para que possam realizar o reconhecimento, atendimento e tratamento precoce de tais agravos para que minimizem os danos e garantindo um bom prognóstico a essas vítimas ${ }^{29}$.

O presente estudo possui como principal limitação a dificuldade de coletar os dados nos boletins de ocorrência impressos do SAMU 192 - ES. Ademais, trata-se de um estudo transversal, o que, portanto, não permite afirmar relações de causa-efeito entre as variáveis associadas. Entretanto, destaca-se como positivo o fato de que, apesar de haver um grande número de trabalhos na literatura referente a quedas em idosos, poucos abordam o tema na área da assistência pré-hospitalar. Dessa forma, destacase a importância de estudos que abranjam este elo da assistência, de forma a gerar melhorias no atendimento aos idosos vítimas de quedas e de outros traumas, considerando-se que um primeiro atendimento adequado e em tempo hábil pode reduzir 
custos e minimizar agravos potenciais à essa população.

\section{Conclusão}

A prevalência de quedas foi de $14,6 \%$, sendo a queda da própria altura a mais comum, sugerindo que mecanismos fisiológicos e posturais estejam envolvidos na sua causa. Observou-se que os principais fatores associados às quedas foram menor risco na gravidade real, gravidade estimada amarela, envio de suporte básico e transporte para serviço de saúde.

\section{Referências}

1. Llompart-Pou J, Pérez-Bárcena J, ChicoFernández M, Sánchez-Casado M, Raurich J. Severe trauma in the geriatric population. World Journal of Critical Care Medicine. 2017; $6 \quad$ (2): $99-106 . \quad$ DOI: http://dx.doi.org/10.5492/wjccm.v6.i2.99

2. Abreu DROM, Novaes ES, de Oliveira RR, Mathias TAF, Marcon SS. Internação e mortalidade por quedas em idosos no Brasil: análise de tendência. Ciência \& Saúde Coletiva. 2018; 23 (4): 1131-41. DOI: http://dx.doi.org/10.1590/1413-

81232018234.09962016.

3. Assis SG, Deslandes SF, Mynaio MCS. Atendimento a pessoas em situação de violência pelo Sistema Único de Saúde. In: Souza Mynaio MC, Gonçalves de Assis S. Novas e Velhas Faces da Violência no Século XXI: visão da literatura brasileira do campo da saúde. $22^{\underline{a}}$ ed. Rio de Janeiro: Editora Fiocruz; 2017. p. 271-95.

4. Martins IR, Silva TKR, Vieira LF, Neto CD, Machado WC, Sogame LCM. Mapeamento das causas externas atendidas pelo SAMU 192/ES de acordo com os ciclos de vida. In: SOGAME, Luciana Carrupt Machado et al (org.). Geotecnologias no serviço de atendimento móvel de urgência e emergência no Espírito Santo: mapeamento para política pública e tomada de decisão. Vitória: Editora Emescam, 2020; Cap. 5. p. 73-87.

5. Minayo MCS, de Souza ER, da Silva MM, de Assis SG. Institucionalização do tema da violência no SUS: avanços e desafios. Ciência \& Saúde Coletiva. 2018; 23 (6): 2007-16. DOI: https://doi.org/10.1590/1413-

81232018236.04962018.

6. Akyama N, Shiroiwa T, Fukuda T, Murashima $S$, Hayashida K. Healthcare costs for the elderly in Japan: Analysis of medical care and long-term care claim records. PloS ONE. 2018; 13 (5): 1-15. DOI: https://doi.org/10.1371/journal.pone.0190392.

7. Dhalwani NN, Fahami $R$, Sathanapally $H$, Seidu S, Davies MJ, Khunti K. Association between polypharmacy and falls in older adults: a longitudinal study from England. BMJ $\begin{array}{llll}\text { Open. } \quad 2017 ; & 7 & \text { (10): } 1-8 . & \text { DOI: }\end{array}$ http://dx.doi.org/10.1136/bmjopen-2017016358.

8. da Silveira FJ, de Oliveira VSL, Friedrich FO, Heinzmann-Filho JP. Hospitalizations and hospital costs due to falls in Brazilian elderly. Scientia Medica, 2020; 30 (1): e36751. DOI: https://doi.org/10.15448/1980-

6108.2020.1.35751.

9. Franck DBP, Costa YCN, Alves KR, Moreira TR, Sanhudo NF, Almeida GBS et al. Trauma em idosos atendidos no serviço de atendimento móvel de urgência. Acta Paul Enferm. 2021; 34: eAPE03081.

10. da Costa RR, França CN, Rodrigues CL, Colombo-Souza P. Fatores de risco associados a quedas em idosos que influenciam o planejamento de assistência ao idoso institucionalizado. Revista Rede de Cuidados em Saúde. 2017; 11 (1): 1-11.

11. Brasil. Ministério da Saúde. Portaria oㅡ 1010, de 21 de maio de 2012. Redefine as Diretrizes Para A Implantação do Serviço de Atendimento Móvel de Urgência (SAMU 192) e Sua Central de Regulação das Urgências, Componente da Rede de Atenção às Urgências. Diário Oficial da União. 22 mai 2012; Seção 1.

12. Secretaria de Atenção à Saúde. Departamento de Atenção Especializada. Regulação Médica das Urgências. Regulação médica das urgências. Brasília: Ministério da Saúde; 2006.

13. Miranda GM, Mendes ACG, da Silva ALA. Population aging in Brazil: current and future social challenges and consequences. Revista Brasileira de Geriatria e Gerontologia. 2016; 19 (3): 507-19. DOI: http://dx.doi.org/10.1590/180998232016019.150140

14. Esteves BB, Pinheiro FS, Carvalho GR, Renó LF, Dornela LL, Dias AMN et al. Incidence of 
elderly accidents attended by Samu in Juiz de Fora - MG. Brazilian Journal Of Health Review. 2021; 2 (4): 8725-8743. DOI: http://dx.doi.org/10.34119/bjhrv4n2-386.

15. Moreland B, Kakara R, Henry A. Tendências em quedas não fatais e lesões relacionadas a quedas entre adultos com idade $\geq 65$ anos Estados Unidos, 2012-2018. MMWR Morb Mortal Wkly Rep 2020; 69: 875-881. DOI: http://dx.doi.org/10.15585/mmwr.mm6927a5

16. Duarte, GP, Ferreira JL, Lebrão ML, Duarte YAO. Relação de quedas em idosos e os componentes de fragilidade. Revista Brasileira de Epidemiologia. 2018; 2 (21): e180017. DOI: https://doi.org/10.1590/1980549720180017.supl.2.

17. Franklin TA, Santos HCS, Junior JAS, Vilela ABA. Caracterização do atendimento de um serviço pré-hospitalar a idosos envolvidos em queda. Revista Online de Pesquisa: Cuidado é Fundamental. 2018; 10 (1): 62-67. DOI: https://doi.org/10.9789/2175-

5361.2018.v10i1.62-67

18. Guerra HS, Sousa RA, Bernardes DCF, Santana JA, Barreira LM. Prevalência de quedas em idosos na comunidade. Revista Saúde e Pesquisa. 2016; 9 (3): 547-55. DOI: https://doi.org/10.17765/2176-

9206.2016v9n3p547-555

19. Gale CR, Cooper C, Sayer AA. Prevalence and risk factors for falls in older men and women: The English Longitudinal Study of Ageing. Age and Ageing. 2016; 45 (6): 789-94. DOI: http://dx.doi.org/10.1093/ageing/afw129

20. Bhangu J, King-Kallimanis BL, Donoghue OA, Carroll L, Kenny RA. Falls, non-accidental falls and syncope in community-dwelling adults aged 50 years and older: Implications for cardiovascular assessment. Plos One. 2017; 12 (7): 1-12. DOI: https://doi.org/10.1371/journal.pone.0180997

21. Prato SCF, de Andrade SM, Cabrera MAS, Dip RM, dos Santos HG, Dellaroza MSG et al. Frequency and factors associated with falls in adults aged 55 years or more. Revista de Saúde Pública. 2017; 51: 1-11. DOI: https://doi.org/10.1590/s15188787.2017051005409

22. Fhon JRS, Rodrigues RAP, Neira WF, Huayta VMR, Robazzi MLCC. Fall and its association with the frailty syndrome in the elderly: systematic review with meta-analysis. Revista da Escola de Enfermagem da Usp. 2016; 50
(6): 1005-13. DOI: https://doi.org/10.1590/s0080623420160000700018

23. Leitão SM, de Oliveira SC, Rolim LR, de Carvalho RP, Filho JMC, Junior AAP. Epidemiologia das quedas entre idosos no Brasil: uma revisão integrativa de literatura. Geriatrics, Gerontology And Aging. 2018; 12 (3): $\quad$ 172-79. DOI: http://dx.doi.org/10.5327/Z2447211520181800030

24. Tiensoli SD, dos Santos ML, Moreira AD, Corrêa AR, Gomes FSL. Características dos idosos atendidos em um pronto-socorro em decorrência de queda. Revista Gaúcha de Enfermagem. 2019; 40 (1): 1-14. DOI: https://doi.org/10.1590/19831447.2019.20180285

25. Leitão SM, Oliveira SC, Rolim LR, Carvalho RP, Coelho Filho JM, Peixoto Junior AA. Epidemiologia das quedas entre idosos no Brasil: uma revisão integrativa de literatura. Geriatrics, Gerontology And Aging. 2018; (12) 3: 172-179. DOI: http://dx.doi.org/10.5327/z2447211520181800030

26. IBGE - Instituto Brasileiro de Geografia e Estatística. Sinopse do Censo Demográfico: 2010. Rio de Janeiro; 2011.

27. da Silva HC, Pessoa RL, de Menezes RMP. Trauma in elderly people: access to the health system through pre-hospital care. Revista Latino-americana de Enfermagem. 2016; 24: 1-8. DOI: https://doi.org/10.1590/15188345.0959.2690.

28. da Costa ACC, Fortes RC. Idosos vítimas de quedas admitidos na terapia intensiva: um estudo retrospectivo analítico. Geriatrics, Gerontology And Aging. 2017; 10 (4): 189-95. DOI: $\quad$ https://doi.org/10.5327/Z2447211520161600043

29. Vieira LF, Neto CD, Sogame LCM. Violências e acidentes nos idosos assistidos no atendimento pré-hospitalar no Espírito Santo, Brasil. Textos \& Contextos. 2019; 18 (1): 161173. DOI: https://doi.org/10.15448/16779509.2019.1.31148.

\section{Informações sobre o Artigo}

Recebido: 20/12/2021

Aceito: 14/01/2022 
Conflitos de interesse: nenhum.

Agências financiadoras: Fundação de Amparo à

Pesquisa e Inovação do Espírito Santo - Fapes. 